\title{
3-iodothyronamine inhibits apoptosis induced by myocardial ischemia reperfusion via the Akt/FoxO1 signaling pathway
}

\author{
Haiyan Zhou ${ }^{1 \#}$, Lili Mo ${ }^{1,2 \#}$, Niwen Huang ${ }^{3}$, Changchao Zou ${ }^{4}$, Chao Li ${ }^{1}$, Muzhi Lin ${ }^{1}$, Bei Zhang ${ }^{1}$, Bo Wei ${ }^{1}$, \\ Ping $\mathrm{Li}^{1}$, Xiaoyun $\mathrm{Si}^{1}$, Jingjing Chen ${ }^{1}$, Wei $\mathrm{Li}^{1}$, Xingde $\mathrm{Liu}^{1}$, Bailong $\mathrm{Hu}^{5}$ \\ ${ }^{1}$ Department of Cardiovascular Medicine, The Affiliated Hospital of Guizhou Medical University, Guiyang, China; ${ }^{2}$ Department of Cardiovascular \\ Medicine, Guizhou Qiannan People's Hospital, Duyun, China; ${ }^{3}$ Department of Respiratory, The Affiliated Hospital of Guizhou Medical University, \\ Guiyang, China; ${ }^{4}$ Department of Cardiovascular Medicine, The Second Affiliated Hospital of Guizhou University of Traditional Chinese Medicine, \\ Guiyang, China; ${ }^{5}$ Department of Anesthesiology, The Affiliated Hospital of Guizhou Medical University, Guiyang, China \\ Contributions: (I) Conception and design: W Li, X Liu, H Zhou, B Hu; (II) Administrative support: W Li, X Liu; (III) Provision of study materials \\ or patients: H Zhou, N Huang, B Wei; (IV) Collection and assembly of data: H Zhou, C Zou, C Li, M Lin, B Zhang, P Li; (V) Data analysis and \\ interpretation: H Zhou, B Hu, L Mo, X Si, J Chen; (VI) Manuscript writing: All authors; (VII) Final approval of manuscript: All authors. \\ \#These authors contributed equally to this work. \\ Correspondence to: Haiyan Zhou; Wei Li; Xingde Liu; Bailong Hu. No. 28 Guiyi Street, Yunyan District, Guiyang 550004, China. \\ Email: zhouhaiyan12388@126.com; liwei249188@sina.com; 1xd@gmc.edu.cn; 375896605@qq.com.
}

Background: This study investigated the potential effects of 3-iodothyronamine (T1AM) on myocardial ischemia reperfusion injury (MIRI) and the underlying molecular mechanisms.

Methods: A total of 16 adult male Sprague-Dawley rats were randomly divided into 4 groups and administered the following: control [60\% dimethyl sulfoxide (DMSO) and 40\% saline, pH 7.4], T1AM (25 mg/kg), T1AM (50 mg/kg), or T1AM (100 mg/kg). The rectal temperatures of the rats were measured at different time points. A further 30 adult male Sprague-Dawley rats were randomized and divided into the following 3 groups ( $\mathrm{n}=10$ in each group): sham operation, ischemia/reperfusion (I/R), and I/R + T1AM. In the I/R and I/R + T1AM groups, the left anterior descending (LAD) coronary artery of the rats were occluded for 0.5 hour to induce myocardial ischemia, followed by reperfusion for 3 hours in the I/R group. The electrocardiography (ECG), cardiac function, and 2,3,5-triphenyltetrazolium chloride (TTC) staining were examined in rats to evaluate the myocardial injury. The differences in the expression of apoptosisrelated and Akt-FoxO1 signaling-related proteins were determined via Western blot.

Results: This work verified that T1AM reduced the body temperature of rats in a dose-dependent manner. Additionally, T1AM improved cardiac function and decreased the infarction size caused by MIRI. T1AM reduced the expression of biochemical parameters and apoptosis of myocardial cells. In addition, after treatment with T1AM, the expression of Glut1, pFoxO1 and Akt were reduced, while the expression of FoxO1 and PPAR $\alpha$ were increased significantly.

Conclusions: Pretreatment of cardiomyocytes with T1AM inhibited apoptosis and protected against ischemia reperfusion injury via the Akt/FoxO1 signaling pathway.

Keywords: Myocardial ischemia reperfusion injury (MIRI); apoptosis; Akt/FoxO1

Submitted Dec 07, 2021. Accepted for publication Feb 14, 2022.

doi: 10.21037/atm-21-7041

View this article at: https://dx.doi.org/10.21037/atm-21-7041 


\section{Introduction}

Ischemic heart disease is a leading cause of morbidity and mortality worldwide (1). Anti-thrombotic therapy and intervention are the main strategies to protect cardiomyocytes against hypoxia-induced damage (2). Previous studies have confirmed that myocardial ischemia reperfusion injury (MIRI) induces cardiomyocyte apoptosis and injury, causing irreversible damage to the heart $(3,4)$. Thus, the development of effective therapeutic strategies that can reduce cardiomyocyte apoptosis and damage is crucial in patients with MIRI (5).

3 -iodothyronamine (T1AM) is an endogenous thyroid hormone derivative with opposing biological effects to those of thyroid hormones (6). Administration of T1AM can lead to a rapid reduction in body temperature, body metabolism $(7,8)$, and heart rate (9). Furthermore, it has therapeutic potential for the treatment of metabolic (10), cardiovascular $(11,12)$, and neurological diseases $(13,14)$. Our previous RNA-sequencing data and in vitro myocardiocytes model demonstrated that T1AM protects cardiomyocytes against hypoxia/reoxygenation (H/R) induced cell injury in AC16 cells via regulation of the FoxO signaling pathway (15). Other studies have shown that administration of T1AM in the isolated heart limited MIRI (16). However, whether T1AM protects the heart from MIRI in rats remains unclear.

This investigation explored the concentration of T1AM needed to reduce the body temperature in rats. The protective effects of T1AM on myocardial ischemia reperfusion-induced cell apoptosis was assessed and the role of the Akt/FoxO1 pathway was examined. The data presented here confirmed that T1AM can attenuate MIRI in vivo via regulation of the $\mathrm{Akt} / \mathrm{FoxO} 1$ pathway.

We present the following article in accordance with the ARRIVE reporting checklist (available at https://atm. amegroups.com/article/view/10.21037/atm-21-7041/rc).

\section{Methods}

\section{Animals}

A total of 46 adult male Sprague-Dawley rats, weighing 200-250 g, were purchased from the Chongqing Tengxin Bill Animals Sales Co., Ltd. [License No: scxk (Qian) 20180001]. All rats were housed in a temperature-controlled environment with a 12:12 hour light-dark cycle, with free access to food and water. After the experiments, the rats were sacrificed with an overdose intravenous injection of
$10 \%$ chloral hydrate at $3 \mathrm{~mL} / \mathrm{kg}$. Animal experiments were approved by the Animal Experimentation Committee of the Institutional Review Board of Guizhou Medical University (License No. 1702046) and complied with the guidelines of Guizhou Medical University for the care and use of animals.

\section{Rectal temperature measurements}

T1AM was dissolved in 60\% dimethyl sulfoxide (DMSO) and physiological saline ( $\mathrm{pH}$ 7.4). On the test day, before drug administration, basal body weights and rectal temperatures (THM 100, Indus Instruments, USA) were measured. Mice were then administered intraperitoneal (i.p) injections of T1AM $(25,50$ or $100 \mathrm{mg} / \mathrm{kg})$ or vehicle $(60 \%$ DMSO and $40 \%$ saline, $\mathrm{pH}$ 7.4). Rectal temperatures were measured at $0.25,0.5,1,1.5,2,3,4,5$, and 6 hours after the injection. All studies were conducted between 09:30 and $15: 30$ in a room with an ambient temperature of $20-21^{\circ} \mathrm{C}$.

\section{Myocardial ischemia reperfusion rat model}

A left thoracotomy was performed in the fourth intercostal space of the rats under pentobarbital sodium ( $40 \mathrm{mg} / \mathrm{kg}$ i.p.) anesthesia. To induce myocardial ischemia, the left anterior descending (LAD) coronary artery was occluded with a 6-0 polypropylene suture $2 \mathrm{~mm}$ below the level of the left atrial appendage. A successful LAD ligation will lead to a pale appearance in the ischemic myocardium. MIRI was induced by ligating the LAD artery for 30 minutes followed by reperfusion for 3 hours by removal of the suture. A total of 30 adult male Sprague-Dawley rats were randomly assigned to three experimental groups, using the random number table method, as follows: (I) the sham group in which rats were subjected to the same procedure but without occlusion of the coronary artery $(\mathrm{n}=10)$; (II) the ischemia/reperfusion (I/R) group, where rats underwent ischemia reperfusion and administration of saline $(\mathrm{n}=10)$; and (III) the I/R + T1AM group, where I/R rats were given $50 \mathrm{mg} / \mathrm{kg}$ T1AM i.p. $(\mathrm{n}=10)$.

\section{Assessment of the size of the myocardial infarct}

2,3,5-triphenyltetrazolium chloride (TTC) staining was performed to determine the infarct size after ischemia reperfusion injury. After 3 hours of reperfusion, rats were sacrificed and hearts were excised and cut into five $2 \mathrm{~mm}^{3}$ slices. For TTC staining, the heart was incubated in $2 \%$ TTC for 15 minutes at $37^{\circ} \mathrm{C}$, and then kept in $4 \%$ 
paraformaldehyde (PFA) prior to acquiring images. The infarcted tissue stains a characteristic white color, whereas the viable tissue stains red. Myocardial IS (left ventricular infarct area as a percentage of the total left ventricular area) was measured using the Image J software.

\section{Western blot analysis}

Total protein was extracted from the rat heart tissues using M-PER mammalian Protein Extraction Reagent (Thermo Scientifics, USA). Protein lysates were boiled for 10 minutes and loaded onto a $10 \%$ sodium dodecyl sulphate-polyacrylamide gel electrophoresis (SDS-PAGE) gel. Proteins were transferred to polyvinylidene fluoride (PVDF) membranes and blocked with 5\% non-fat milk. Membranes were then incubated overnight at $4{ }^{\circ} \mathrm{C}$ with primary antibodies against PPAR $\alpha$ (Abcam, UK), FoxO1 (Abcam, UK), phosphorylated FoxO1 (Cst, USA), Akt (Cst, USA), Gck (Abcam, UK), Glut1 (Abcam, UK), Bcl-2 (Abcam, UK), or Bax (Proteintech, China). After washing with Tris buffered saline Tween (TBST), membranes were incubated with secondary antibodies (1:10,000, Proteintech, China) for 1 hour at room temperature (RT), washed again with TBST, and visualized with enhanced chemiluminescence reagents. Chemiluminescence images were obtained by using the ChemiDoc Imaging Systems (Bio-rad) and Image J software was used to quantify protein expression.

\section{Assessment of myocardial injury}

After the rat hearts underwent 240 minutes of reperfusion, blood samples were collected and analyzed. Plasma creatine kinase (CK), CK-MB, and lactate dehydrogenase $(\mathrm{LDH})$ were analyzed with an automatic biochemistry analyzer (Watford Olympus Au640, UK), according to manufacturer's instruction.

\section{Transmission electron microscopy (TEM)}

The ultrastructure of the cardiac muscles and mitochondrial were observed by TEM. Briefly, the myocardial tissues were cut into $1 \mathrm{~mm}^{3}$ pieces and fixed with $4 \%$ glutaraldehyde overnight. Subsequently, the tissue was fixed with $2 \%$ osmic acid at RT for 2 hours. A graded series of ethanol solutions were used to dehydrate the tissue. The samples were then transferred to a mixture of propylene oxide and embedding solution $(10 \mathrm{~mL})$ at RT for 1 hour. After staining with uranium acetate and lead citrate, myocardial tissues were observed using a TEM (JEM-2000EX TEM, Japan).

\section{Echocardiographic analysis}

Echocardiographic measurements were performed at 3 hours after the induction of myocardial infarction (MI). The rats were anesthetized with pentobarbital sodium (40 mg/kg i.p.) and placed in a supine position. Electrocardiography (ECG) measurements were performed in all rats.

Echocardiographic parameters such as left ventricular ejection fraction (LVEF) and left ventricular fractional shortening (LVFS) were recorded. Three consecutive cardiac cycles were analyzed and the average was used for data analysis. The time points selected for echocardiographic measurements were pre-experiment (baseline), myocardial ischemia, and 3 hours after myocardial ischemia.

\section{Identification of active targets}

All pharmacological targets of active compounds were screened and collected from the Swiss target prediction database (https://www.swisstargetprediction.ch), Batman, and DrugBank. The repetitive genes were identified after data correction and the gene names were further extracted using the UniProt database.

\section{Identification of myocardial ischemia reperfusion-associated genes}

The study was conducted in accordance with the Declaration of Helsinki (as revised in 2013).

Genes related to myocardial ischemia reperfusion were selected from Genecards (https://www.genecards.org/), Online Mendelian Inheritance in Man (OMIM) databases (https://www.omim.org/), and NCBI gene module and Therapeutic Target Database (TTD, http://db.idrblab.net/ $\mathrm{ttd} /$ ). The intersection of the retrieved targets of T1AM and myocardial ischemia reperfusion-related genes were obtained for downstream analysis.

\section{Gene Ontology (GO) and Kyoto Encyclopedia of Genes and Genomes (KEGG) patbway enrichment analysis}

The overlapping genes of T1AM and myocardial ischemia reperfusion were retrieved for GO and KEGG enrichment analysis using the DAVID database (https://david.ncifcrf. 


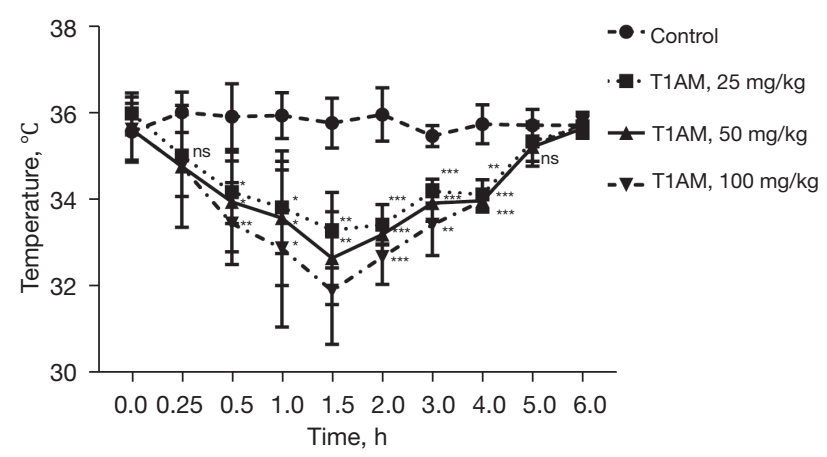

Figure 1 The rectal temperature of rats at different time points. The effects of T1AM injected intraperitoneally on rectal temperature in male rats. T1AM at 25,50 , and $100 \mathrm{mg} / \mathrm{kg}(\mathrm{n}=4)$ significantly reduced $(\mathrm{P}<0.05)$ the rectal temperature which returned to baseline after $6-7$ hours. ${ }^{*}, \mathrm{P}<0.05$; ${ }^{* *}, \mathrm{P}<0.01$; ***, $\mathrm{P}<0.005$; ns, not significant. T1AM, 3-iodothyronamine.

gov/) (17) with a $\mathrm{P}$ value $>0.05$ as the criteria. GO enrichment analysis included biological processes (BPs), molecular functions, cellular components, and KEGG pathway analysis were performed using "ClusterProfiler", "GOplot" and "org.Hs.eg.Db".

\section{Identifying the essential targets of T1AM against myocardial ischemia reperfusion}

All intersecting targets of T1AM and myocardial ischemia reperfusion-related genes were input into the STRING database to obtain the protein-protein interaction (PPI) network interaction. Cytoscape software (Version 3.6.1) was applied for visualization of Compound-Target GenesDisease network.

\section{Molecular docking}

The 3D structure of disease-related genes was obtained from the Protein Data Bank (PDB) database. The 3D structure of active compounds and disease-related genes were input into Autodock Vina (Version 1.1.2) for molecular docking analysis. The conformation with the best affinity was visualized in Pymol2.3.

\section{Statistical analysis}

All statistical analyses were performed using SPSS 20.0 software (IBM SPSS Software). Continuous data are presented as means \pm standard deviation $(\mathrm{SD})$ when the data followed a normal distribution. Differences between two groups were studied using Student's $t$-test or one-way analysis of variance (ANOVA) for multiple groups. A P value $<0.05$ was considered to statistically significant.

\section{Results}

\section{Hypothermia in rats treated with T1AM}

Intraperitoneal injection of T1AM into SD rats reduced the body temperature within 6 hours, with the basal body temperature decreasing by $3-4{ }^{\circ} \mathrm{C}$ after exposure to $50 \mathrm{mg} / \mathrm{kg}$ T1AM. The rats became inactive but reflexes were retained. At 2 hours post-injection, gradual reversal of the hypothermia effects was observed and the body temperature of the rats returned to baseline after 6-7 hours (Figure 1).

\section{T1AM preconditioning limited the MI area}

MIRI was induced in rats as depicted in the schematic diagram shown in Figure $2 A$ and confirmed via electrocardiography (Figure $2 B$ ). The area of MI, expression of myocardial injury markers, cardiac function, and morphological changes in the myocardial tissues were assessed. During the 3 hours of ischemia, $3.33 \pm 0.57$ rats experienced ventricular fibrillation $(\mathrm{VF})$ in the $\mathrm{I} / \mathrm{R}$ group and $2 \pm 1$ rats experienced $\mathrm{VF}$ in the T1AM treated group (Figure S1). However, the incidence of VF was not significantly different between the 2 groups. TTC staining showed that treatment with T1AM at dose of $50 \mathrm{mg} / \mathrm{kg}$ significantly decreased the size of the $\mathrm{MI}$ compared to untreated I/R rats $(\mathrm{P}<0.001$; Figure $2 C, 2 D)$.

\section{T1AM preconditioning alleviated MIRI in rats}

Echocardiography was performed to determine whether T1AM treatment affected cardiac function. I/R rats treated with T1AM developed significantly less adverse remodeling, as well as better ejection fraction (EF) and fractional shortening (FS) at 3 hours after myocardial ischemia compared to $\mathrm{I} / \mathrm{R}$ rats with no treatment $(\mathrm{P}<0.001$; Figure $3 A-3 C$ ). The T1AM treated I/R rats also had better $\mathrm{LV}$ end systolic pressure, left ventricular end systolic volume (LVESV), left ventricular end-diastolic volume (LVEDV), $\mathrm{dP} / \mathrm{dt}_{\max }$, and $\mathrm{dP} / \mathrm{dt}_{\min }$ values compared to $\mathrm{I} / \mathrm{R}$ rats with no treatment $(\mathrm{P}<0.05)$ (Figure $\mathrm{S} 1$ ), suggesting improved cardiac hemodynamic responses after treatment 
A

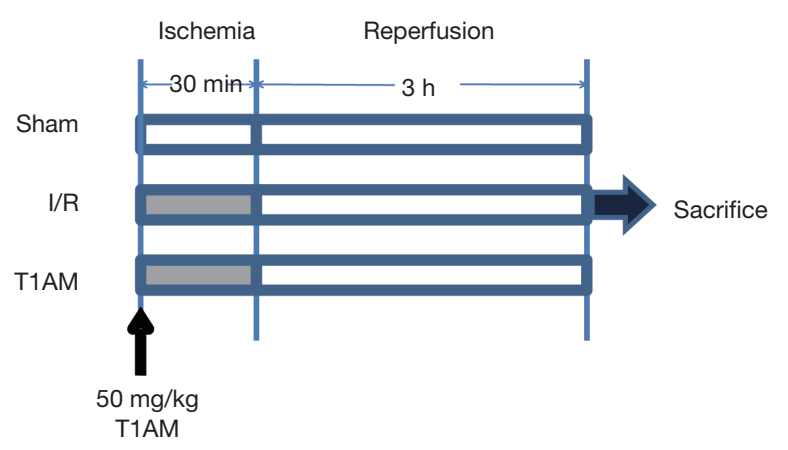

B

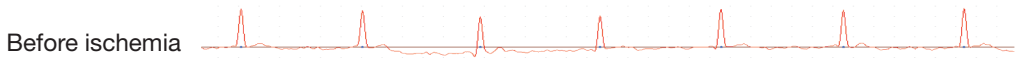

Ischemia

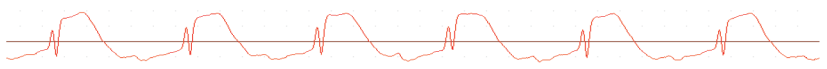

Reperfusion

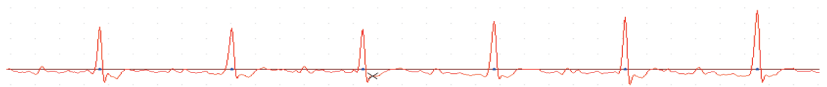

C
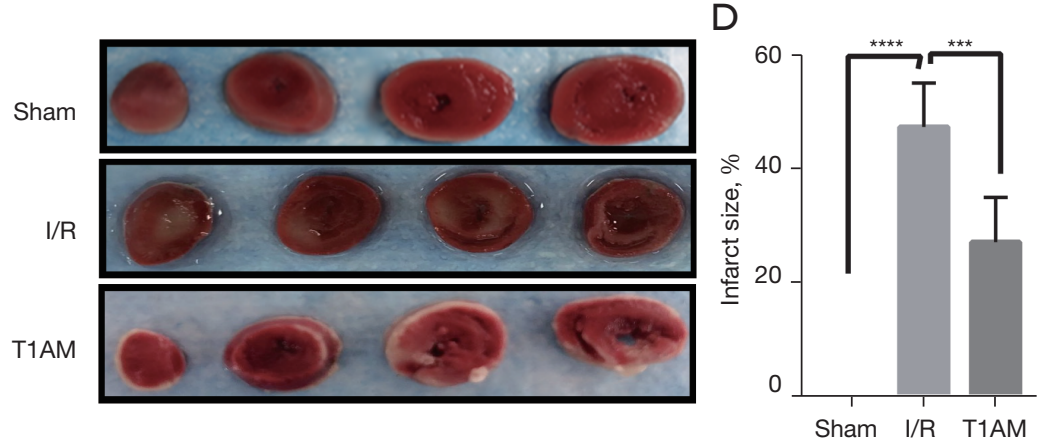

Figure 2 T1AM preconditioning limited the myocardial infarction area. (A) Schematic representation of the myocardial ischemia reperfusion procedure. (B) Representative electrocardiographs before ischemia, during myocardial ischemia, and after reperfusion. (C) Representative images of TTC staining. (D) Quantitative analysis of the infarct size. ${ }^{* * *}, \mathrm{P}<0.005$; ${ }^{* * * *}, \mathrm{P}<0.001$. I/R, ischemia/reperfusion; T1AM, 3-iodothyronamine; TTC, 2,3,5-triphenyltetrazolium chloride.

with T1AM.

To further verify the protective role of T1AM in myocardial I/R injury, the ultrastructure of cardiomyocytes was observed using TEM. Cardiomyocytes from the I/R rats showed obvious mitochondrial disruption, dissolution, and irregular cardiac muscle fibers. In comparison, T1AM treated $\mathrm{I} / \mathrm{R}$ rats showed suppressed mitochondria dissolution and improved cardiac muscle fibers (Figure 3D). The expression of cardiomyocyte injury biochemical markers including LDH, CK, and CK-MB were significantly increased in I/R rats, while treatment with T1AM decreased LDH, CK, and CK-MB levels compared with the I/R group (Figure 3E-3G).

\section{Identifying the T1AM targets that intersect with myocardial ischemia reperfusion}

The Network Pharmacology database identified 638 genes associated with myocardial ischemia reperfusion from the Genecards, OMIM, Therapeutic Target Database, and NCBI databases (Figure 4A). A total of 100 genes were identified as T1AM-associated targets through the Swiss Target Prediction. An overlap of the myocardial ischemia reperfusion genes with the $\mathrm{T} 1 \mathrm{AM}$-associated targets 
A
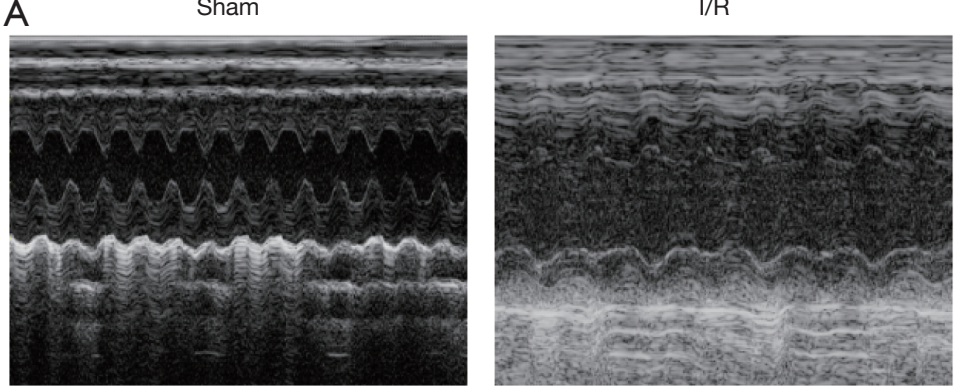

C

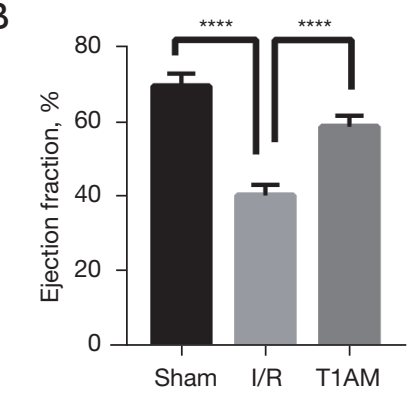

D

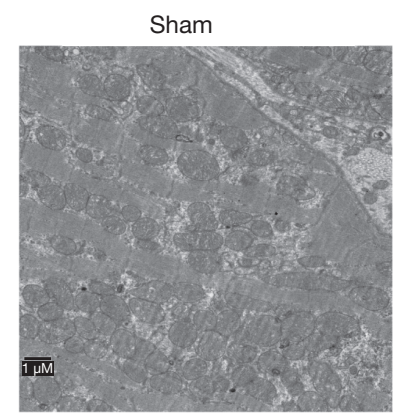

E

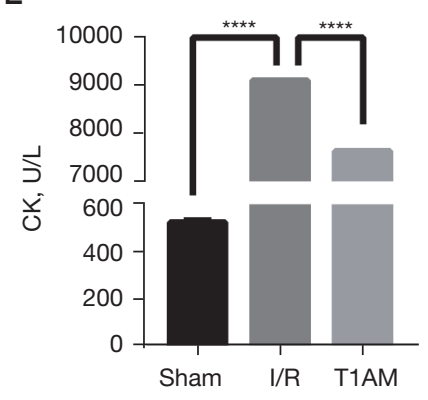

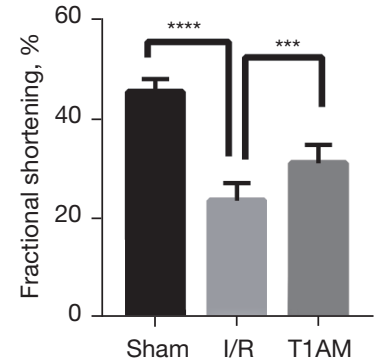

I/R

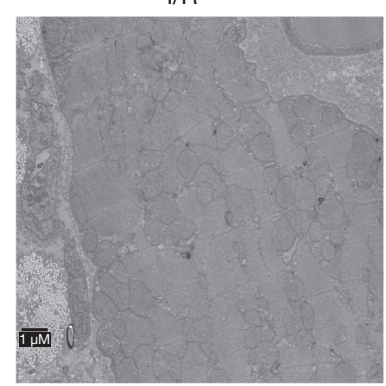

$\mathrm{F}$

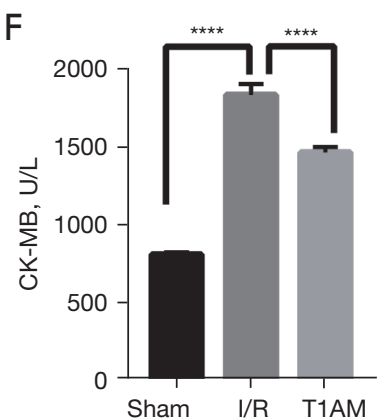

T1AM
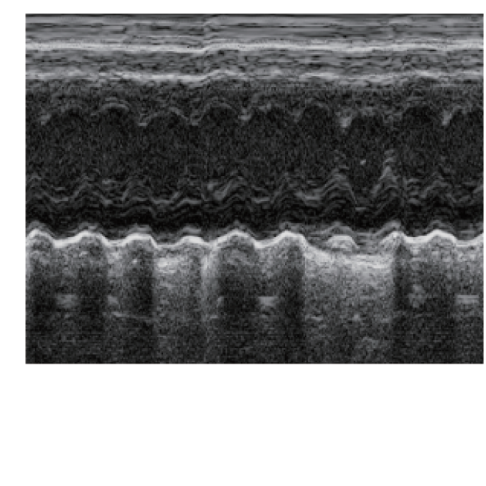
A

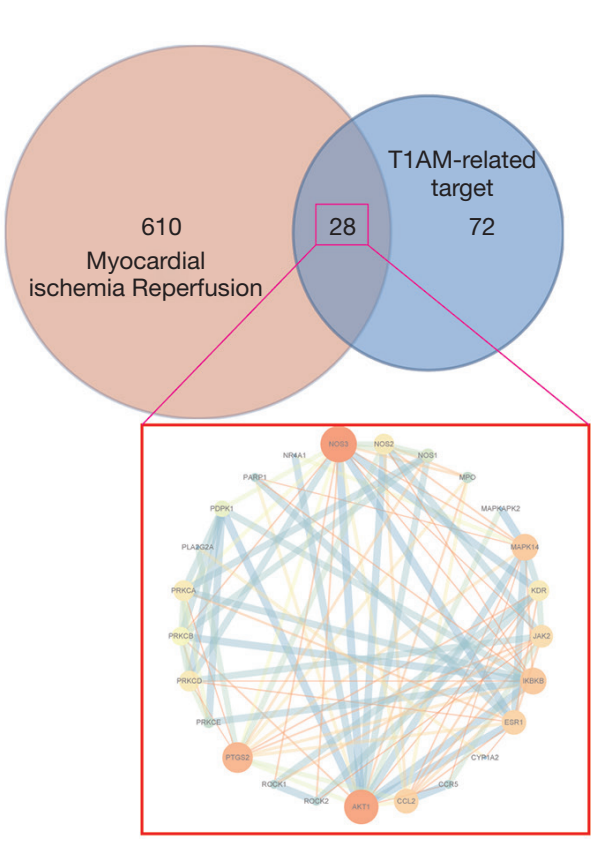

B

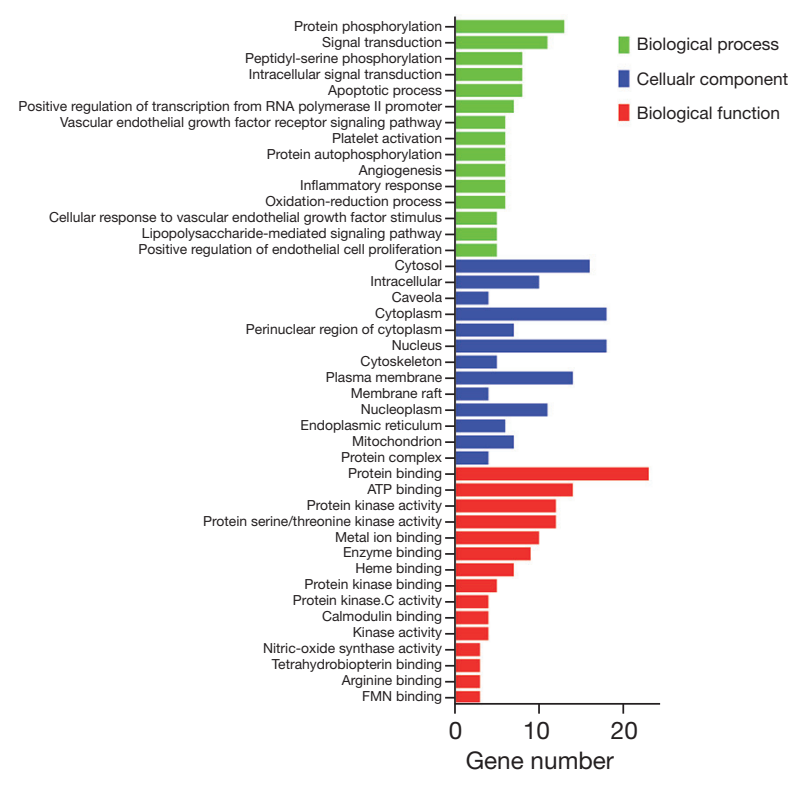

C

D
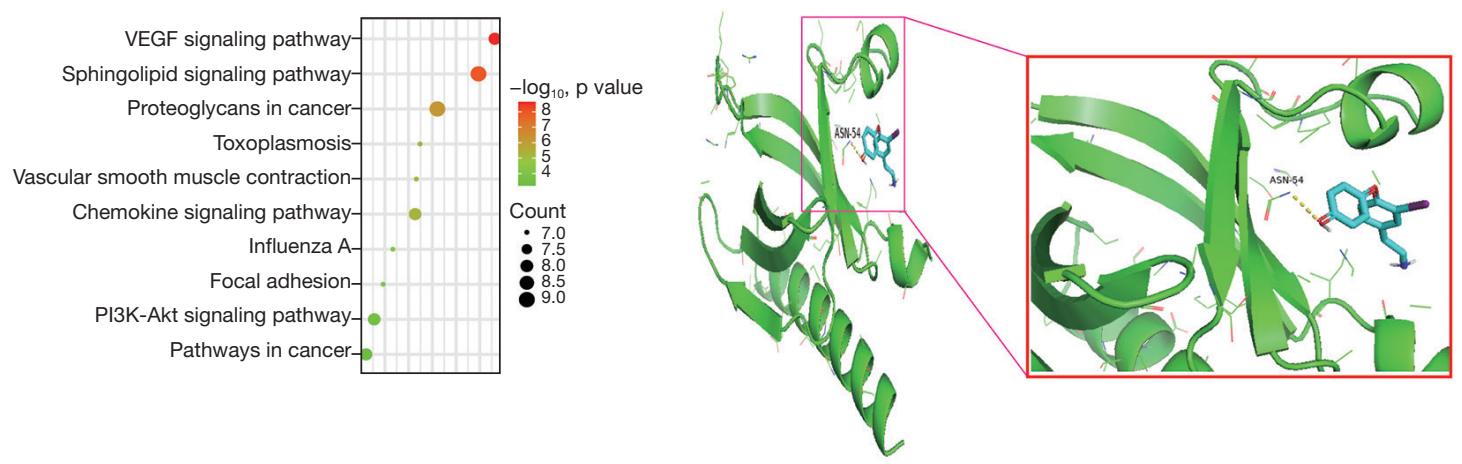

$\mathrm{E}$
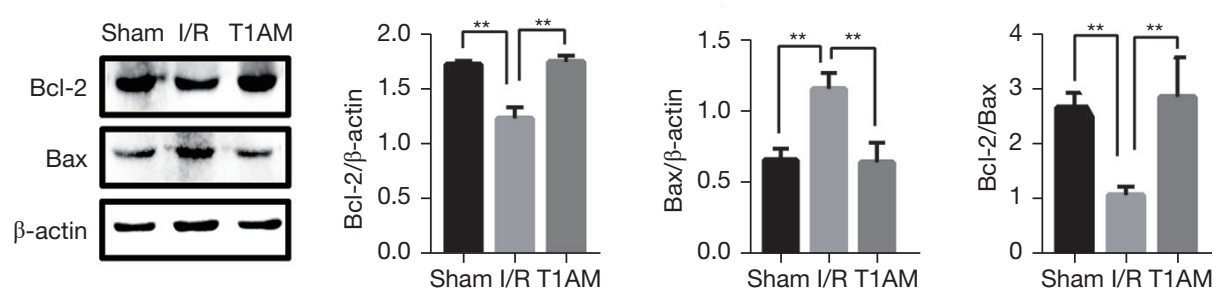

Figure 4 Pretreatment with T1AM suppressed apoptosis after myocardial ischemia/reperfusion injury. (A) Venn diagram depicting the intersecting genes of T1AM and myocardial ischemia reperfusion. (B) Gene ontology analysis of the intersecting genes of T1AM and myocardial ischemia reperfusion. (C) KEGG pathway of intersecting genes of T1AM and myocardial ischemia reperfusion. (D) Binding of T1AM and Akt using molecular docking. (E) Western blot detection of Bcl-2 and Bax. ${ }^{* *}, \mathrm{P}<0.01 . \mathrm{I} / \mathrm{R}$, ischemia/reperfusion; T1AM, 3-iodothyronamine; KEGG, Kyoto Encyclopedia of Genes and Genomes. 
identified 28 intersecting genes.

\section{Identification of the main targets of T1AM against myocardial ischemia reperfusion and molecular docking}

To further explore the intersecting genes, STRING analysis was performed to obtain the PPI network mediated by the 28 intersecting genes of T1AM against myocardial ischemia reperfusion (Figure $4 A$ ). GO enrichment analysis demonstrated that T1AM was involved in a series of BPs including apoptosis, signal transduction, and protein phosphorylation. Based on the analysis of cellular components, the targets included the cytosol, intracellular space, and caveola. Furthermore, associated molecular functions mainly included protein binding, ATP binding, and protein kinase activity (Figure 4B).

KEGG enrichment revealed that the intersecting genes related to the phosphatidylinositol-3-kinase (PI3K)Akt signaling pathway, the vascular endothelial growth factor (VEGF) signaling pathway, and the chemokine signaling pathway (Figure 4C). The Akt-T1AM docking $(-5.6 \mathrm{kcal} / \mathrm{mol})$ is shown in Figure $4 D$. The active cavity box parameter setting center $\mathrm{x}, \mathrm{y}, \mathrm{z}$ were $21.707,14.463$, and 9.91, respectively, while the size $\mathrm{x}, \mathrm{y}, \mathrm{z}$ were 80,84 , 126, respectively. This suggested that T1AM showed good binding activity with the Akt protein.

\section{T1AM preconditioning suppressed apoptosis after myocardial I/R injury}

To further investigate the detailed role of T1AM in alleviating myocardial I/R injury, the levels of Bcl-2 and Bax expression in heart tissues were examined by Western blot analysis (Figure 4E). T1AM treatment increased the expression of the anti-apoptotic protein $\mathrm{Bcl}-2$, reduced the expression of the pro-apoptotic protein Bax, and increased the proportion of $\mathrm{Bcl} 2 / \mathrm{Bax}$ in $\mathrm{I} / \mathrm{R}$ rats compared to $\mathrm{I} / \mathrm{R}$ rats with no treatment.

\section{T1AM preconditioning alleviated cardiomyocytes apoptosis,} which is partially dependent on Akt/FOXO1 activation

Our previous studies showed that the Akt/FoxO1 pathway plays an important role in MIRI $(12,14)$. Therefore, the molecular mechanisms involved in T1AM-medicated cardio-protection and the effects of T1AM on the Akt/FoxO1 pathway were investigated. As shown in Figure 5, Western blot analysis demonstrated that I/R injury increased pFoxO1 and Glut1 expression and reduced FoxO1 and PPAR $\alpha$ expression. Administration of T1AM reduced the expression of Glut1, pFoxO1 and Akt, but increased the expression of FoxO1 and PPAR $\alpha$.

\section{Discussion}

The present study identified T1AM as a protective agent against myocardial ischemia reperfusion. These findings revealed the following: (I) administration of T1AM can lead to a rapid reduction in the body temperature of rats; (II) T1AM can attenuate the cardiac injury by reducing the infarct area, improving cardiac function, reducing the incidence of arrhythmias, altering the expression of biochemical markers, and inhibiting the apoptosis of cardiomyocytes; and (III) T1AM may reduce the apoptosis of cardiomyocytes via $\mathrm{Akt} / \mathrm{FoxO} 1$ pathway.

Our previous study examined the effects of T1AM on H/ R-induced AC-16 cells. Based on both RNA-seq and cellbased assays, T1AM was shown to mediate hypometabolism and exert protective effects on cardiomyocytes via regulation of the FoxO signaling pathway $(12,15)$. However, limited studies have reported the protective role of T1AM in vivo. Myocardial ischemia reperfusion can lead to lethal ventricular arrhythmias (18). The current data showed that T1AM decreased the incidence of VF, suggesting that T1AM can attenuate MIRI. Furthermore, the expression levels of LDH, CK, and CK-MB, which are biochemical markers of cardiomyocytes injury and clinically used as diagnostic marker of MI, were significantly reduced in the T1AM group.

Echocardiography can efficiently identify reginal wall motion abnormalities in MI (19). In this study, echocardiography was used to evaluate the cardiac function in rats as reflected by FS and EF. At 3 hours posted myocardial ischemia reperfusion, treatment with T1AM was shown to significantly increase cardiac function, including $\mathrm{EF}$ and FS, compared to I/R rats with no treatment. Additionally, TTC and hemodynamic results were in agreement with the cardiac function results.

MIRI triggers cell apoptosis which deteriorates heart function (17). Previous studies have reported that proteins such as $\mathrm{Bax}$ and $\mathrm{Bcl} 2$ control the intrinsic apoptosis pathway (20). Of note, the ratio of Bcl-2 and Bax plays a critical role in the cell apoptosis process (21). Pretreatment with T1AM significantly reduced the expression of Bax and increased the expression of Bcl-2, thus elevating the Bcl-2/ Bax ratio. This data confirmed that T1AM preconditioning 

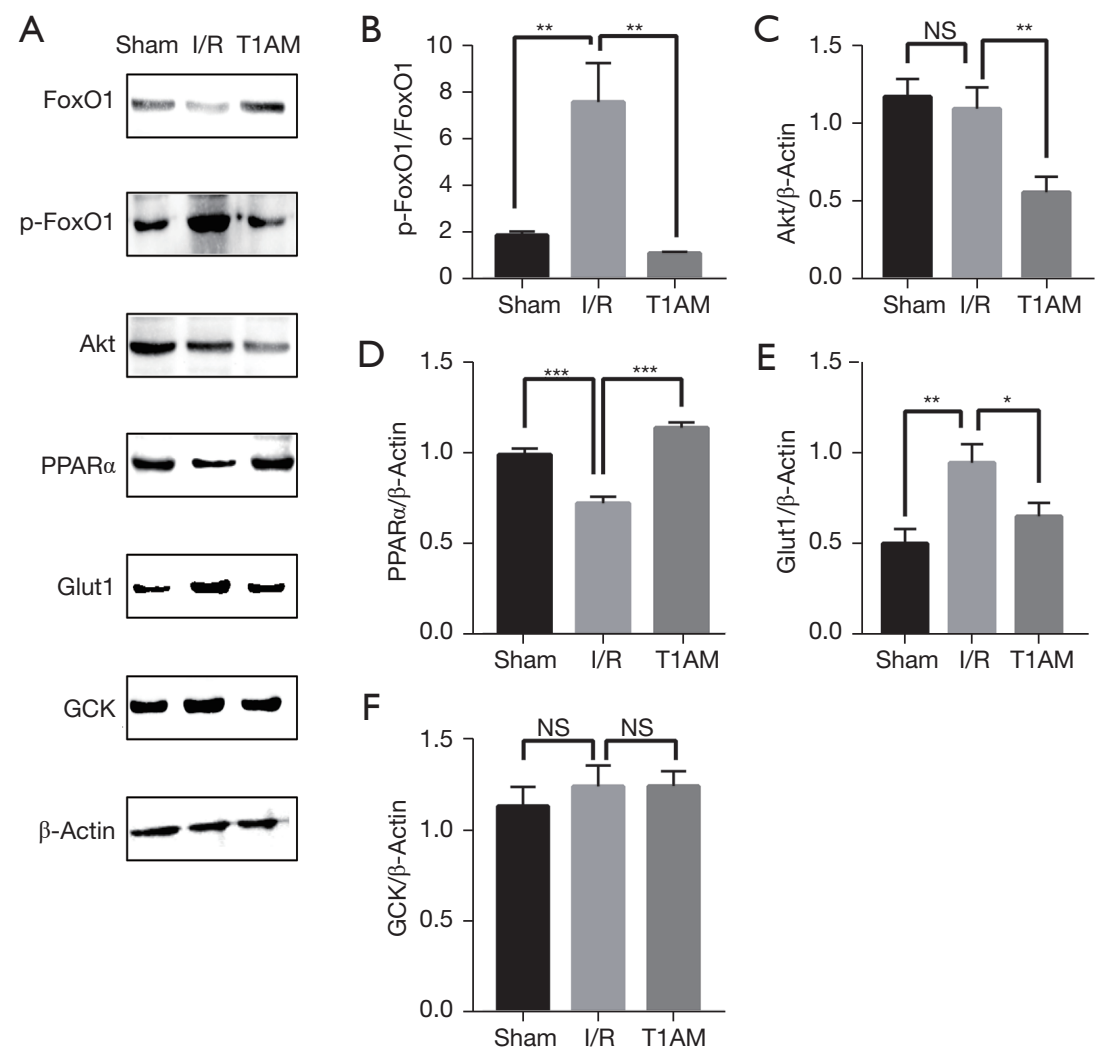

Figure 5 Pretreatment of T1AM suppressed apoptosis via the Akt/FoxO signaling pathway. (A) Western blot detection of FoxO1, pFoxO1, Akt, PPAR $\alpha$, Glut1, and GCK. (B) Quantification of pFoxO1. (C) Quantification of Akt. (D) Quantification of PPAR . (E) Quantification of Glut1. (F) Quantification of GCK. Data are presented as mean $\pm \mathrm{SD}$. * $\mathrm{P}<0.05$; **, $\mathrm{P}<0.01$; **, $\mathrm{P}<0.005$; I/R, ischemia/reperfusion; T1AM, 3-iodothyronamine; FoxO1, forkhead box protein O1; pFoxO1, phosphorylated FoxO1; PPAR $\alpha$, peroxisome proliferator-activated receptor $\alpha$; Glut1, glucose transporter 1; GCK, glucokinase; NS, not significant; SD, standard deviation.

reduced cellular apoptosis induced by MIRI, thereby exerting a protective effect on the cardiomyocytes.

The Akt/FoxO1 axis mediates multiple biological functions that are crucial for cellular differentiation, including metabolic reprogramming (22), apoptosis (23), oxidative stress response, and DNA damage repair (24). Previous studies revealed that FoxO1 inhibition improved mitochondrial biogenesis and suppressed mitophagy. Expression of PPAR was downregulated in both renal I/ $\mathrm{R}$ and H/R injury, which could be abrogated by FOXO1 inhibition (25). In our previous research, inhibition of FoxO1 and PPAR $\alpha$ disrupted cell metabolism, resulting in cardiomyocyte apoptosis (15). T1AM is a promising compound that regulates cellular metabolic reprogramming (26). Here, we demonstrated that T1AM significantly reduced the expression of Glut1, $\mathrm{pFoxO} 1$, and Akt, but increased the expression of FoxO1 and PPAR $\alpha$ expression, which may represent a potential mechanism of suppression of cell apoptosis. A limitation of our results is that T1AM was performed in vivo with pretreatment. More concentrations of T1AM without pretreatment should be performed to confirm the interesting and promising results obtained here.

\section{Conclusions}

This report demonstrated that pretreatment of T1AM improved cardiac function in response to ischemia reperfusion injury through regulating the expression of apoptotic proteins. Exogenous T1AM preconditioning suppressed I/R-induced cell apoptosis, which may be partially mediated through the Akt/FoxO1 pathway. Therefore, T1AM may be a potential therapeutic agent for the treatment of patients with MIRI. 


\section{Acknowledgments}

Funding: This work was supported in part by grants from the National Natural Science Foundation of China (Nos. 81904319, 82160951, 82060855, 31760294, 82160086, and 81960047); the Science and Technology Fund of Guizhou Provincial Health Department [qiankehejichu(2018)1137, qiankehejichu(2019)1260, qiankehezhicheng(2021)yb063, qiankehejichu(2020)1Y298]; the Fund of Guiyang Science and Technology Department [(2021)43-6, (2019)9-1-13]; the Health and Family Planning Commission of Guizhou Province (gzwkj2021-106, gzwkj2021-273); and the PhD Early Development Program of the Affiliated Hospital of Guizhou Medical University (gyfybsky-2021-33).

\section{Footnote}

Reporting Checklist: The authors have completed the ARRIVE reporting checklist. Available at https://atm. amegroups.com/article/view/10.21037/atm-21-7041/rc

Data Sharing Statement: Available at https://atm.amegroups. com/article/view/10.21037/atm-21-7041/dss

Conflicts of Interest: All authors have completed the ICMJE uniform disclosure form (available at https://atm. amegroups.com/article/view/10.21037/atm-21-7041/coif). The authors have no conflicts of interest to declare.

Ethical Statement: The authors are accountable for all aspects of the work in ensuring that questions related to the accuracy or integrity of any part of the work are appropriately investigated and resolved. Animal experiments were approved by the Animal Experimentation Committee of the Institutional Review Board of Guizhou Medical University (License No. 1702046) and complied with the guidelines of Guizhou Medical University for the care and use of animals. The study was conducted in accordance with the Declaration of Helsinki (as revised in 2013).

Open Access Statement: This is an Open Access article distributed in accordance with the Creative Commons Attribution-NonCommercial-NoDerivs 4.0 International License (CC BY-NC-ND 4.0), which permits the noncommercial replication and distribution of the article with the strict proviso that no changes or edits are made and the original work is properly cited (including links to both the formal publication through the relevant DOI and the license).
See: https://creativecommons.org/licenses/by-nc-nd/4.0/.

\section{References}

1. Dong Y, Chen H, Gao J, et al. Molecular machinery and interplay of apoptosis and autophagy in coronary heart disease. J Mol Cell Cardiol 2019;136:27-41.

2. Harky A, Maskell P, Burgess M. Anti-platelet and anticoagulant therapy in peripheral arterial disease prior to surgical intervention. Vascular 2019;27:299-311.

3. Zheng Y, Shi B, Ma M, et al. The novel relationship between Sirt3 and autophagy in myocardial ischemiareperfusion. J Cell Physiol 2019;234:5488-95.

4. Xu W, Zhang L, Ma S, et al. TRAF5 protects against myocardial ischemia reperfusion injury via AKT signaling. Eur J Pharmacol 2020;878:173092.

5. Otaka N, Shibata R, Ohashi K, et al. Myonectin Is an Exercise-Induced Myokine That Protects the Heart From Ischemia-Reperfusion Injury. Circ Res 2018;123:1326-38.

6. Köhrle J, Biebermann H. 3-Iodothyronamine-A Thyroid Hormone Metabolite With Distinct Target Profiles and Mode of Action. Endocr Rev 2019;40:602-30.

7. Zucchi R, Accorroni A, Chiellini G. Update on 3-iodothyronamine and its neurological and metabolic actions. Front Physiol 2014;5:402.

8. Zucchi R, Rutigliano G, Saponaro F. Novel thyroid hormones. Endocrine 2019;66:95-104.

9. Gencarelli M, Laurino A, Landucci E, et al. 3-Iodothyronamine Affects Thermogenic Substrates' Mobilization in Brown Adipocytes. Biology (Basel) 2020;9:95.

10. Rutigliano G, Bandini L, Sestito S, et al. 3-Iodothyronamine and Derivatives: New Allies Against Metabolic Syndrome? Int J Mol Sci 2020;21:2005.

11. Axelband F, Dias J, Ferrão FM, et al. Nongenomic signaling pathways triggered by thyroid hormones and their metabolite 3-iodothyronamine on the cardiovascular system. J Cell Physiol 2011;226:21-8.

12. Haiyan Z, Bailong H, Bei Z, et al. Comparative Transcriptome Analysis Reveals the Potential Cardiovascular Protective Targets of the Thyroid Hormone Metabolite 3-Iodothyronamine (3-T1AM). Biomed Res Int 2020;2020:1302453.

13. Laurino A, Lucenteforte E, De Siena G, et al. The impact of scopolamine pretreatment on 3-iodothyronamine (T1AM) effects on memory and pain in mice. Horm Behav 2017;94:93-6.

14. Doyle KP, Suchland KL, Ciesielski TM, et al. 
Novel thyroxine derivatives, thyronamine and

3-iodothyronamine, induce transient hypothermia and marked neuroprotection against stroke injury. Stroke 2007;38:2569-76.

15. Zhou H, Hu B, Liu X. Thyroid Hormone Metabolite 3-Iodothyronamine (T1AM) Alleviates Hypoxia/ Reoxygenation-Induced Cardiac Myocyte Apoptosis via Akt/FoxO1 Pathway. Med Sci Monit 2020;26:e923195.

16. Frascarelli S, Ghelardoni S, Chiellini G, et al. Cardioprotective effect of 3-iodothyronamine in perfused rat heart subjected to ischemia and reperfusion. Cardiovasc Drugs Ther 2011;25:307-13.

17. Li J, Cheng R, Wan H. Overexpression of TGR5 alleviates myocardial ischemia/reperfusion injury via AKT/GSK-3 $\beta$ mediated inflammation and mitochondrial pathway. Biosci Rep 2020;40:BSR20193482.

18. Ghiasi R, Mohammadi M, Majidinia M, et al. The effects of mebudipine on myocardial arrhythmia induced by ischemia-reperfusion injury in isolated rat heart. Cell Mol Biol (Noisy-le-grand) 2016;62:15-20.

19. Tomoaia R, Beyer RS, Simu G, et al. Understanding the role of echocardiography in remodeling after acute myocardial infarction and development of heart failure with preserved ejection fraction. Med Ultrason 2019;21:69-76.

20. Malsy M, Bitzinger D, Graf B, et al. Staurosporine induces apoptosis in pancreatic carcinoma cells PaTu 8988t and

Cite this article as: Zhou $\mathrm{H}$, Mo L, Huang N, Zou C, Li C, Lin M, Zhang B, Wei B, Li P, Si X, Chen J, Li W, Liu X, Hu B. 3-iodothyronamine inhibits apoptosis induced by myocardial ischemia reperfusion via the $\mathrm{Akt} / \mathrm{FoxO} 1$ signaling pathway. Ann Transl Med 2022;10(4):168. doi: 10.21037/atm-21-7041
Panc-1 via the intrinsic signaling pathway. Eur J Med Res 2019;24:5.

21. Ouhtit A, Gupta I, Gaur RL, et al. Deregulation of cell growth and apoptosis in $\mathrm{UV}$-induced melanomagenesis. Front Biosci (Elite Ed) 2020;12:223-36.

22. Yan K, Da TT, Bian ZH, et al. Multi-omics analysis identifies FoxO1 as a regulator of macrophage function through metabolic reprogramming. Cell Death Dis 2020;11:800.

23. Zhang $M$, Zhang Q, Hu Y, et al. miR-181a increases FoxO1 acetylation and promotes granulosa cell apoptosis via SIRT1 downregulation. Cell Death Dis 2017;8:e3088.

24. Xing YQ, Li A, Yang Y, et al. The regulation of FOXO1 and its role in disease progression. Life Sci 2018;193:124-31.

25. Wang D, Wang Y, Zou X, et al. FOXO1 inhibition prevents renal ischemia-reperfusion injury via cAMPresponse element binding protein/PPAR- $\gamma$ coactivator$1 \alpha$-mediated mitochondrial biogenesis. Br J Pharmacol 2020;177:432-48.

26. Bellusci L, Runfola M, Carnicelli V, et al. Endogenous 3-Iodothyronamine (T1AM) and Synthetic Thyronamine-like Analog SG-2 Act as Novel Pleiotropic Neuroprotective Agents Through the Modulation of SIRT6. Molecules 2020;25:1054.

(English Language Editor: J. Teoh) 

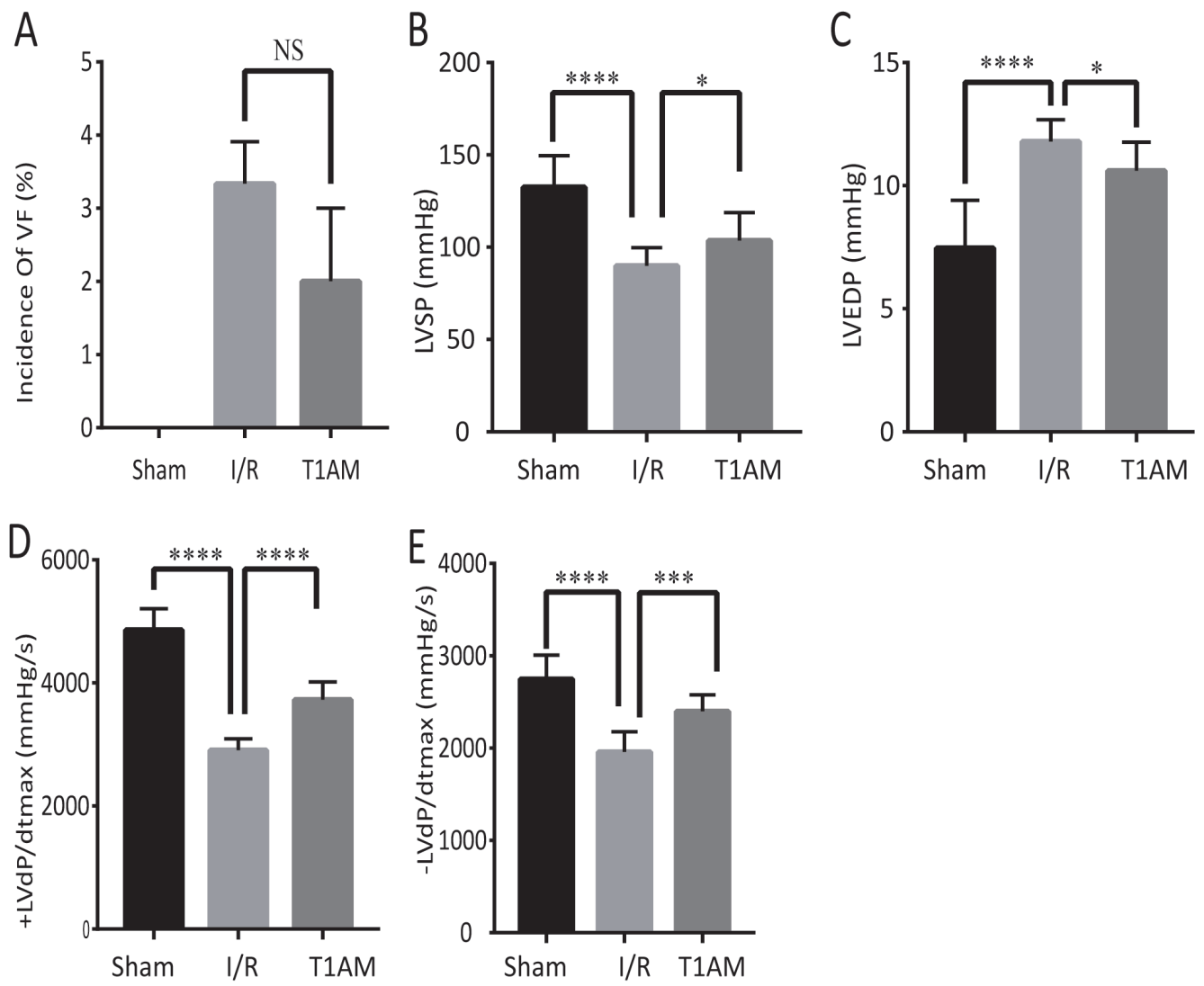

Figure S1 T1AM improved cardiac hemodynamic responses after myocardial ischemia/reperfusion injury. (A) Incidence of VF. (B-E) T1AM improves rat cardiac function after 30 minutes ischemia and 3 hours reperfusion. ${ }^{*}, \mathrm{P}<0.05 ;{ }^{* * *}, \mathrm{P}<0.005 ;{ }^{* * * *}, \mathrm{P}<0.001$. I/R, ischemia/ reperfusion; T1AM, 3-iodothyronamine; VF, ventricular fibrillation; LVSP, left ventricular systolic pressure; LVEDP, left ventricular end diastolic pressure; ${ }_{ \pm} \mathrm{LVdP} / \mathrm{dtmax}$, the instantaneous first derivation of left ventricle pressure; NS, not significant. 